\title{
Also d'Susi wär e ganz e liebi Frau für de Markus! Zur Verdoppelung des indefiniten Artikels in der adverbiell erweiterten Nominalphrase im Schweizerdeutschen
}

\author{
Janine Steiner (Zürich)
}

\begin{abstract}
Three possible constructions of adverbially complemented noun phrases are attested in Swiss German. In (1), the indefinite article is doubled, whereas in (2) and (3) the article appears only once; it precedes or follows the adverb, respectively:

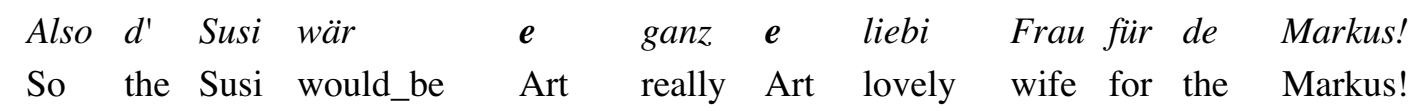

'Susi would be a really lovely wife for Markus!'

$\begin{array}{llllllll}\text { Also } & d^{\prime} & \text { Susi } & \text { wär } & \text { ganz } & \boldsymbol{e} & \text { liebi } & \text { Frau für de } \\ \text { So } & \text { the } & \text { Susi } & \text { would_be } & \text { really } & \text { Art } & \text { lovely } & \text { wife for the }\end{array}$

'Susi would be a really lovely wife for Markus!'

$\begin{array}{llllllll}\text { Also } & d^{\prime} & \text { Susi } & \text { wär } & \boldsymbol{e} & \text { ganz } & \text { liebi } & \text { Frau für de Markus! } \\ \text { So the } & \text { Susi } & \text { would_be } & \text { Art } & \text { really } & \text { lovely } & \text { wife for the Markus! }\end{array}$

'Susi would be a really lovely wife for Markus!'
\end{abstract}

Doubling of the indefinite article is quite often mentioned in dialectological literature. Unfortunately there is nothing to be found about regional differences, frequency or possible contexts for a doubling in most standard works of Swiss German dialectology. The present paper now fills in some of these gaps in research on the adverbially complemented noun phrase in Swiss German:

In a quantitative analysis will be shown that the construction with the article between adverb and adjective (2) is the one given the highest acceptance and preference rates by the informants. Furthermore the paper also shows that the doubling-construction (1) is more prominent in some Swiss German speaking-areas than in others. An apparent time analysis reveals ongoing language change and, last but not least, it shows that sociolinguistic parameters have an impact on the adverbially complemented noun phrase as well.

\section{$1 \quad$ Vorbemerkungen}

Einige finden, dass Susi e ganz e liebi Frau für Markus wäre, während andere sie für ganz e liebi Frau halten. Für dritte wiederum ist sie gar e ganz liebi Frau. - Der vorliegende Beitrag stellt einen Erklärungsversuch dar, der zeigen soll, durch welche Parameter die Konstruktion der adverbiell erweiterten Nominalphrase beeinflusst wird. 
Es gibt im Schweizerdeutschen drei Möglichkeiten, den Sachverhalt, dass Susi eine ganz liebe Frau für Markus wäre, auszudrücken:

(1) Also d'Susi wär e ganz e liebi Frau für de Markus!

(2) Also d'Susi wär ganz e liebi Frau für de Markus!

(3) Also d'Susi wär e ganz liebi Frau für de Markus!

Die Variante (1) ist eine Konstruktion mit doppelt gesetztem Indefinitartikel, während die zweite und dritte Variante den Artikel nur einfach verwenden. Bei der Variante (2) befindet sich der Artikel zwischen der adverbiellen Erweiterung und dem Adjektiv, während er bei der dritten Variante vorangestellt, also vor der adverbiellen Erweiterung und dem Adjektiv steht. Hörbelegen zufolge sind die drei Konstruktionen im Schweizerdeutschen koexistent.

\section{Forschungsstand}

Das Phänomen der Artikelverdoppelung ist in der Sekundärliteratur grundsätzlich bekannt. Leider lässt sich in den meisten Standardwerken zur Dialektologie jedoch kaum etwas zum geographischen Vorkommen, zur Häufigkeit oder zu den Auftretensbedingungen für eine Verdoppelung finden. Einige wenige Ansätze zu einer Entstehungsthese sind vorhanden, jedoch noch nicht empirisch überprüft worden.

In Stalders Versuch eines Schweizerischen Idiotikons von 1806 lässt sich zum Phänomen der Artikelverdoppelung noch nichts finden, während im Idiotikon (1881: 1. Band, 273) im Artikel zu ein folgender Abschnitt steht:

6. [...] Beliebt ist Wiederholung des 'ein' vor einem durch ein Adv. bestimmten Adj. und bei 'so' z. B. en b'sonderig en fria Maa, ein besonders freundlicher Mann AP; e recht e schöni; es recht es grosses $\mathrm{SCHW}$; $\mathrm{S}$ Z; eso e ... solch ein GL, Z.

Das Idiotikon belegt mit diesem Eintrag, dass das Phänomen des verdoppelten indefiniten Artikels Ende des 19. Jahrhunderts bekannt war. Es liefert uns die Information, dass die Verdoppelung in Kombination mit einem durch ein Adverb bestimmten Adjektiv auftritt. Weiter erfahren wir, dass diese Konstruktionsart beliebt sei und anhand der Beispiele auch, in welchen Kantonen diese auftritt, nämlich Appenzell, Schwyz, Solothurn, Zürich und im Falle von eso auch Glarus.

Gustav Binz (1888: 17) schreibt in seinem Buch Zur Syntax der baseldeutschen Mundart im Kapitel Die Adjectiva werden auf verschiedene Weise ergänzt: 
[...] 4. Durch Adverbien: arg, famos, ferterli, ganz, gar, grisli, haillos, kaibemässig, mainaidig, recht u.a. [...] Bei dieser Ergänzung eines Adjectivs durch ein Adverb ist oft eine merkwürdige scheinbare Angleichung der Form des Adverbs an diejenige des Adjectivs eingetreten: e gar e liebe Bueb; ${ }^{1}$ der Lehrer muess e Landma si und zwor e rechte gscheite, aber nur bei masculinen Substantiven. Im Femininum und Neutrum ist diese Angleichung nicht wahrzunehmen: s'ische gar e bravi Frau; mer hen e ganz e schen Hus; e so ne schen Klaid, nie e ganzi bravi Frau etc., so dass ich fast eher geneigt wäre, hier eine Doppelsetzung des Artikels anzunehmen. Ich dächte mir dieselbe in der Art entstanden, dass man in der Zwischenstellung des Artikels zwischen Adverb und Adjectiv, die nur in diesen wenigen Fällen vorkam, den Artikel nicht mehr herausfühlte und ihn nun noch einmal an die sonst übliche Stelle vor das Adverb setzte. Vielleicht liesse sich die Entwickelung auch so denken, dass beim Masculinum ursprünglich eine solche Formangleichung vorlag (wie dies heute noch z. B. im Schwäbischen ganz deutlich der Fall ist), dann aber eine Doppelsetzung des Artikels empfunden wurde, worauf dieselbe nach Analogie der Beispiele beim Masculinum auch beim Femininum und Neutrum eingetreten wäre.

Wie auch das Idiotikon nimmt Binz das Phänomen zur Kenntnis und weitet damit dessen Vorkommensregion auf das Baseldeutsche aus. Er ist sich allerdings nicht sicher, ob es sich tatsächlich um eine Doppelsetzung des Artikels oder um eine Formangleichung zwischen Adjektiv und Adverb handelt. Des Weiteren liefert uns Binz ebenfalls die Information, dass dieses Phänomen in Kombination mit einem durch ein Adverb ergänzten Adjektiv auftritt, und dass dies oft geschieht. Was allerdings fehlt, ist die Angabe, ob diese Formangleichung oder eben Doppelsetzung obligatorisch oder fakultativ ist, ob sie bei allen Adverbien in gleicher Weise möglich ist und nach welchen Regeln sie auftritt.

Arthur Frey behandelt im Jahre 1906 das Phänomen ebenfalls, allerdings weit weniger ausführlich. Im Kapitel zu den Adverbien erwähnt er in einem Nebensatz, dass das gradbestimmende Adverb vor das Adjektiv tritt, worauf eine nochmalige Vorsetzung des Artikels stattfinden kann. Hier erhalten wir nur die elementarste Information, dass das Phänomen des verdoppelten Artikels existiert, jedoch keine weiteren Angaben zu Regelmässigkeit oder in welchen Dialekten es vorkommen kann. Weise (1909) erwähnt die Konstruktion für verschiedene deutsche Mundarten. Auch Stucki (1921) nimmt davon Kenntnis.

In der Zürichdeutschen Grammatik von Albert Weber (1948: 203) lässt sich zu dem Phänomen ebenfalls etwas finden und zwar im Kapitel zu den Adverbien:

Zur Stellung des Adverbs. Gewisse der Steigerung dienende Adverbien stehen nicht unmittelbar vor dem Adjektiv, sondern treten vor den Artikel, der häufig doppelt gesetzt wird.

Man findet jedoch auch hier keine exakten Angaben zu den Umständen, die erforderlich sind, damit der Artikel verdoppelt wird.

In der Luzerndeutschen Grammatik (Fischer 1960), in der Baseldeutsch-Grammatik (Suter 1976) und auch im Zuger Mundartbuch (Bossard 1962) findet man wiederum dieselben Formulierungen wie in der Zürichdeutschen Grammatik.

\footnotetext{
${ }^{1}$ Hier müsste es vom Argumentationszusammenhang her eigentlich $e$ gare liebe Bueb heissen, damit die von Binz erwähnte Formangleichung des Adverbs an das Adjektiv deutlicher illustriert würde.
} 
In Werner Hodlers Berndeutscher Syntax (1969: 708) wird das Phänomen folgendermassen abgehandelt: ${ }^{2}$

Die Antizipation bedingt also die Verschiebung schwachbetonter Satzteile aus ihrer ordentlichen Stellung, so dass sie daselbst vermisst und neu gesetzt werden, also im Satze nun doppelt erscheinen. Das gilt vor allem für den unbestimmten Artikel: e gar e guete Bueb, der vil der besser $W y$. [...]

In der Berndeutsch-Grammatik von Werner Marti (1985) ist über die Konstruktion mit doppelt gesetztem Indefinitartikel nichts zu finden. Auch im Sprachatlas der Deutschen Schweiz (SDS) wird das Phänomen nicht behandelt.

In neueren linguistischen Abhandlungen findet das Phänomen Erwähnung bei Bucheli Berger/Glaser (2002) und Christen (2003) (für das Schweizerdeutsche) und bei HennMemmesheimer (1986) und Plank (2003) (im Zusammenhang mit verschiedenen deutschen Dialekten und mit der Umgangssprache).

Dieser Überblick zur vorhandenen Sekundärliteratur über den Forschungsgegenstand macht deutlich, dass weitere Untersuchungen auf dem Gebiet von grosser Wichtigkeit sind, da einzig die Existenz des Phänomens, jedoch nicht dessen Auftretensbedingungen beschrieben werden.

\section{$3 \quad$ Fragestellung/Datenmaterial}

Das Datenmaterial, welches diesem Beitrag zugrunde liegt, wurde im Rahmen des Nationalfondsprojektes Dialektsyntax des Schweizerdeutschen an der Universität Zürich erhoben. ${ }^{3}$ Dort war im ersten Fragebogen an die Gewährsleute die folgende Frage enthalten:

\footnotetext{
${ }^{2}$ Es handelt sich hier um den ersten Eintrag im Unterkapitel 'Doppelsetzung verschobener Wörter' innerhalb des Kapitels 'Spaltung'.

3 Zu Ziel und Methode des Projektes (NF-Nr. 1114-057121 und NF-Nr. 1113-068244) sowie Ortsnetz und Gewährspersonen cf. Bucheli Berger/Glaser (2002) sowie Bucheli Berger (2005).
} 
Frage 10: Bruno kennt eine nette junge Frau, die er sich durchaus an der Seite seines immer noch ledigen Sohnes Markus vorstellen könnte. Er sagt:

\&elche der folgenden Sätze können Sie in Ihrem Dialekt sagen ("ja"), welche sind nicht möglich ("nein")?

$$
\text { ja nein }
$$

1) $\square$ Also d' Susi wär e ganz e liebi Frau für de Markus!

2) $\square \quad$ Also d' Susi wär ganz e liebi Frau für de Markus!

3) $\quad$ a Also d' Susi wär e ganz liebi Frau für de Markus!

Welche Variante ist für Sie die natürlichste?

$\mathrm{Nr}$.

\& Würden Sie den Satz normalerweise in einer Form sagen, die nicht aufgeführt ist?

$\square$ ja $\square$ nein

Wenn "ja": Bitte notieren Sie hier den Satz so, wie Sie ihn normalerweise sagen würden:

Es handelt sich um eine Frage des Typs Multiple Choice, wobei im Falle des Ankreuzens von mehreren "Ja"-Möglichkeiten gleichzeitig nach der natürlichsten Variante (Präferenz) gefragt wird. Laut Bucheli Berger/Glaser (2002: 18f.) ergibt dieser Fragetyp beim Phänomen der Verdoppelung des indefiniten Artikels die besten Ergebnisse, "since in a translation or completion question, a large number of informants would follow the standard German construction (3) which, in fact, appears in dialect, but very rarely".

Zum Zeitpunkt der Auswertung (Juli 2002) waren 2705 erste Fragebogen retourniert und in einer Datenbank erfasst worden.

\section{$4 \quad$ Zur quantitativen Verbreitung der Konstruktionen}

In einem ersten Schritt soll gezeigt werden, wie die drei möglichen Konstruktionsweisen von den Gewährspersonen quantitativ akzeptiert worden sind. Dabei ergab sich folgende Verteilung: ${ }^{4}$

\footnotetext{
${ }^{4}$ Die aufgeführten Prozentangaben überschreiten zusammengezählt 100\%, da die Gewährspersonen die Möglichkeit hatten, mehrere Varianten mit "Ja" anzukreuzen.
} 


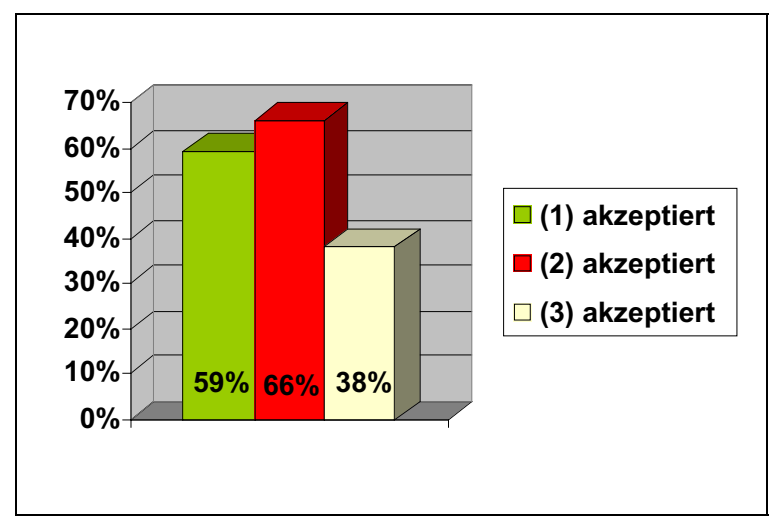

Abb. 1: Akzeptanz der Varianten 1-35

Die grösste Akzeptanz weist die Variante (2) auf, gefolgt von (1). Die dritte Variante ist, wie Bucheli Berger/Glaser (2002) erwähnten, eher selten, allerdings nicht "very rarely", wie dort angenommen.

Betrachtet man nun die Verteilung bei der Zusatzfrage nach der natürlichsten Variante, ergibt sich ein ähnliches Bild wie oben:

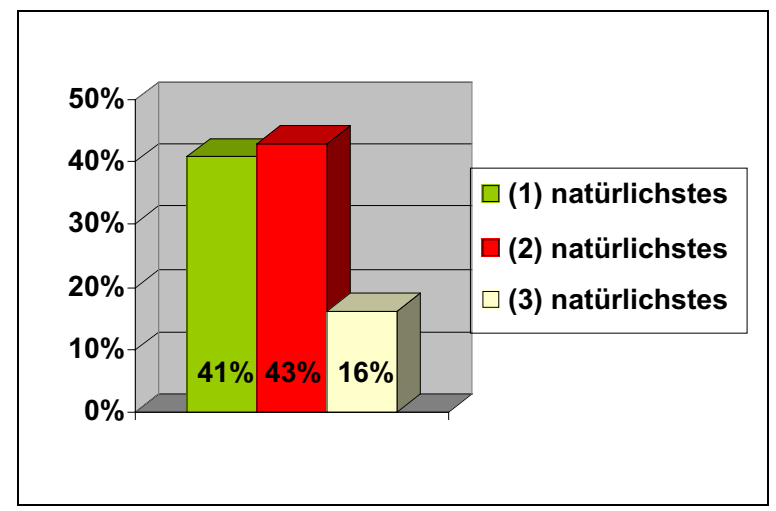

Abb. 2: Natürlichste Variante

Auch hier ist die Variante (2) die am häufigsten genannte, jedoch ist der Abstand zu Variante (1) geringer. Die Variante (3) ist mit nur 16\% selten. Variante (1) wird also von $41 \%$ aller Gewährspersonen präferiert. Von denjenigen Gewährsleuten, welche die Konstruktion generell akzeptieren (cf. oben), bezeichnen sie 69\% als natürlichste Variante. Die Variante (2) wird im Vergleich zu Variante (1) nur von $2 \%$ weniger Gewährspersonen als natürlichste verwendet. Man könnte also sagen, dass die beiden Varianten (1) und (2) fast gleichwertig nebeneinander existieren.

Dies legt nun die Vermutung nahe, dass die beiden quantitativ äquivalenten Konstruktionen (1) und (2) auf zwei verschiedene regionale Gebiete distribuiert sind. Auch für die selten auftretende Variante (3) könnte man die These vertreten, dass sie in einem kleineren, begrenzten Gebiet vorkommt. Im folgenden Kapitel werde ich diesen Hypothesen nachgehen.

\footnotetext{
${ }^{5}$ Die Ziffern (1) bis (3) beziehen sich auf die im Fragebogen suggerierten Varianten (cf. oben).
} 


\section{$5 \quad$ Zur geographischen Verbreitung der Konstruktion}

Im Folgenden konzentriere ich mich auf das Vorkommen der Variante (1) mit dem verdoppelten indefiniten Artikel. In untenstehender Karte ist abgebildet, wo sich die oben erwähnten 59\% der Gewährspersonen, welche bei Variante (1) "Ja" ankreuzten, befinden.

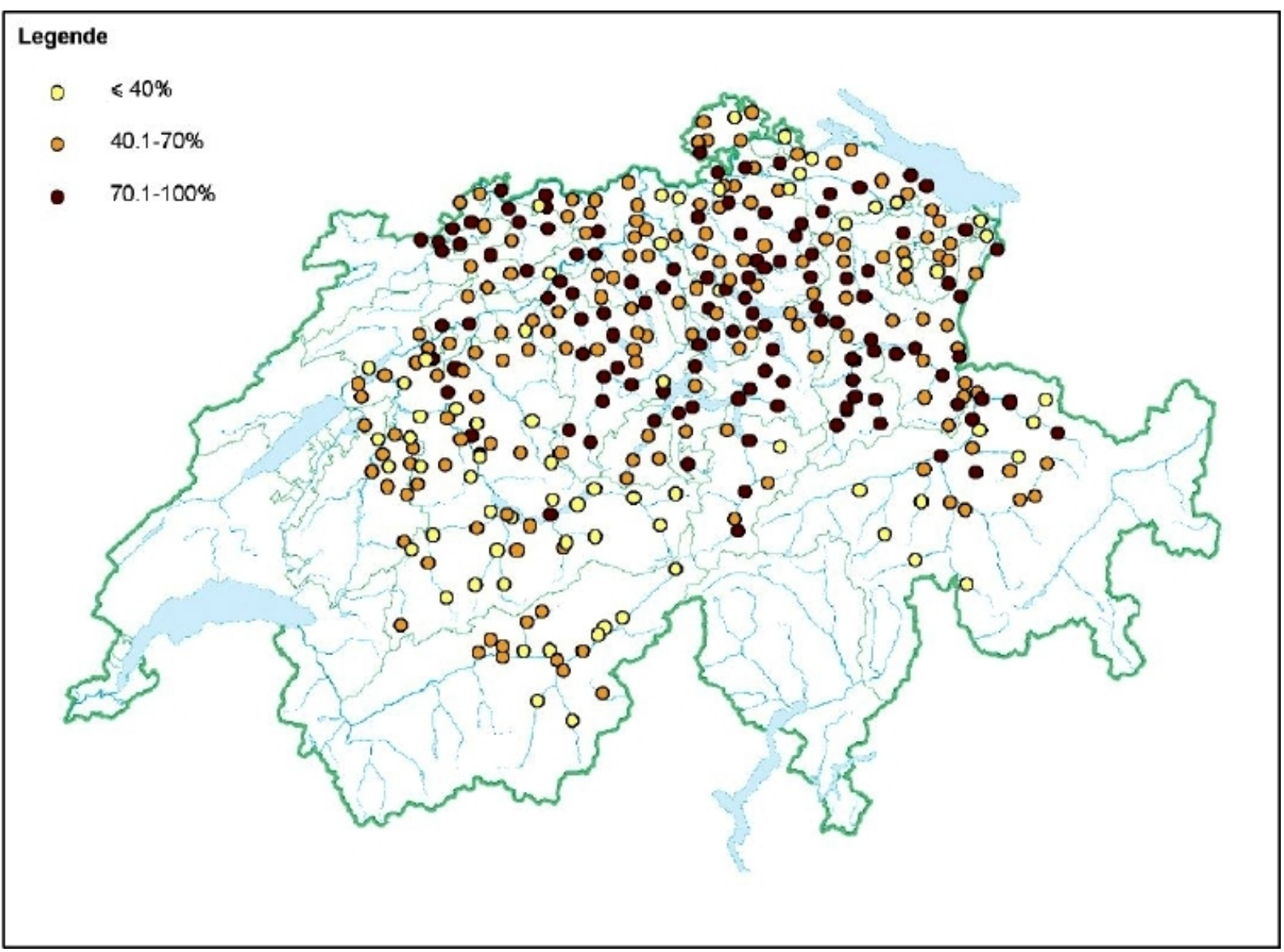

Abb. 3: "Also d' Susi wär e ganz e liebi Frau für de Markus!" - akzeptiert

Auf den ersten Blick erscheint einem das Vorkommen der Variante (1) über das gesamte deutschsprachige Gebiet der Schweiz verteilt. Bei genauerem Hinsehen kann man jedoch erkennen, dass sich die schwarzen Punkte, das sind diejenigen Ortspunkte, bei denen mehr als $70 \%$ aller am Ort befragten Gewährspersonen die Variante (1) mit "Ja" beantwortet haben, vorwiegend im Norden befinden. ${ }^{6}$ Man kann sogar sagen, dass sich ein Gürtel von grosser grosser Akzeptanz, nämlich 70.1-100\%, von Basel über die Innerschweiz bis an den Bodensee und in den Kanton Graubünden erstreckt. In den Kantonen Glarus und Schwyz finden sich fast ausschliesslich schwarze Punkte. Hingegen beobachtet man im Wallis, im Kanton Bern, Freiburg und in den Kantonen Appenzell, Schaffhausen und Graubünden fast nur graue oder weisse Punkte, woraus sich schliessen lässt, dass die Verdoppelung des indefiniten Artikels in diesen Gebieten weniger weit verbreitet ist.

\footnotetext{
${ }^{6}$ Selbstverständlich ist hier bei den Prozentangaben zu berücksichtigen, dass die absolute Gesamtzahl pro Ort relativ niedrig sein kann. Ein einzelner schwarzer Punkt ist daher nicht aussagekräftig ohne Prüfung der Verhältnisse.
} 
Dieser Eindruck wird noch verstärkt, betrachtet man die folgende Karte, aus welcher diejenigen Ortspunkte ersichtlich sind, die Variante (1) als natürlichste bezeichnen.

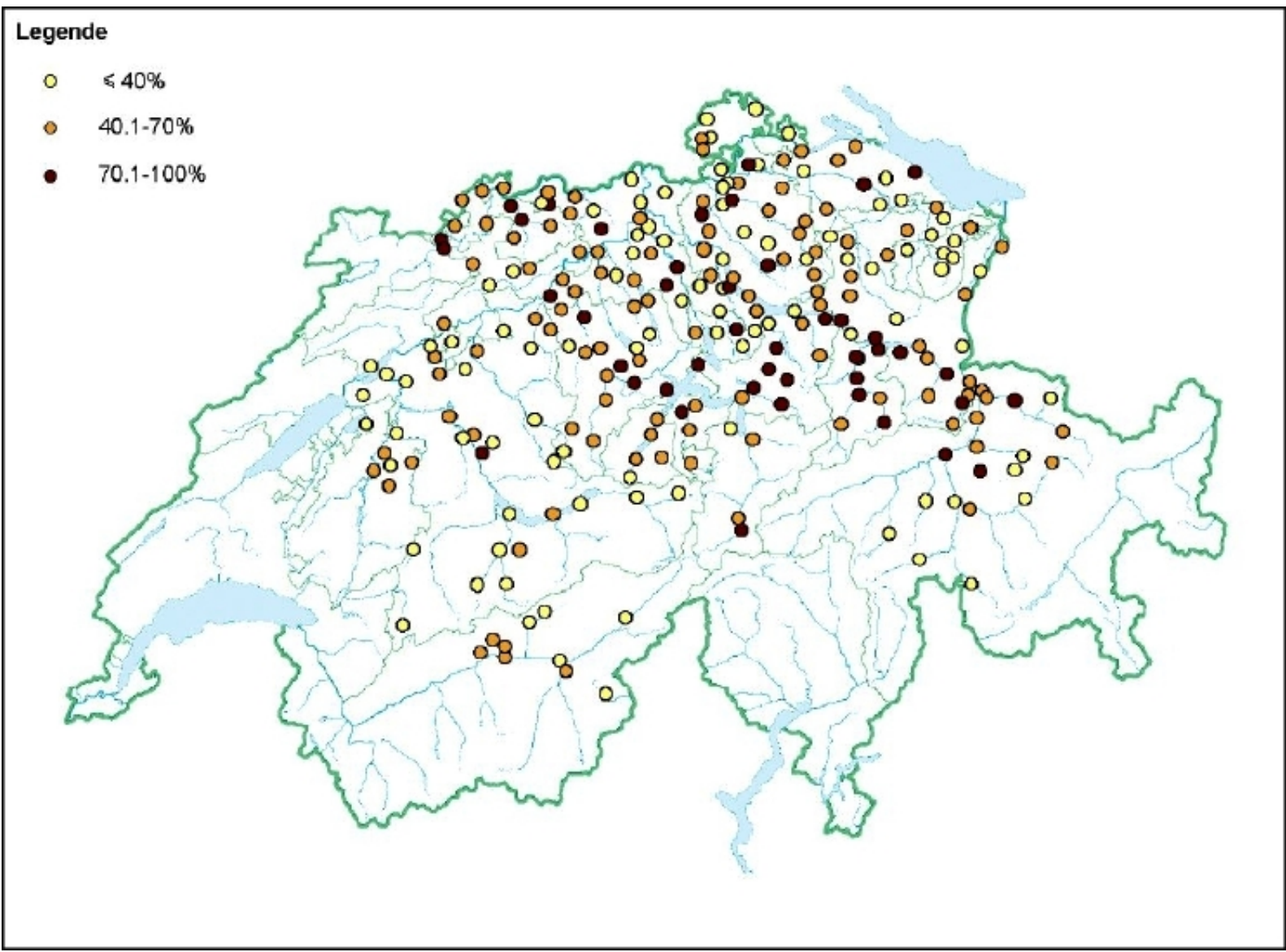

Abb. 4: "Also d' Susi wär e ganz e liebi Frau für de Markus!" - natürlichste Variante

Bei dieser Karte wurden zusätzlich die Einzelnennungen herausgefiltert. Es sind nur Ortspunkte abgebildet, bei welchen mehr als eine Person die Verdoppelung als natürlichste Variante verwendet. Damit kann ich vermeiden, dass bei einzelnen Ortspunkten ein 100\%Wert entsteht, wo lediglich eine Person die Frage beantwortet hat. Zudem interessieren mich im Hinblick auf die mögliche Existenz eines geographischen Kernareals nicht primär Einzelnennungen, sondern diejenigen Gebiete, in welchen hohe Prozentwerte vorhanden sind. Zudem muss man auch bedenken, dass es bei einem so geringen Wert auch möglich sein kann, dass es sich um einen Ausfüllfehler, bedingt z. B. durch Ermüdung, handelt. Würde ich jedoch diese Einzelnennungen in obiger Karte abbilden, entstünde zwar eine Vorkommenskarte mit sämtlichen Belegen, jedoch kein Raumbild.

Die obige Abbildung deutet erneut darauf hin, dass sich das Verdoppelungsgebiet in einem Gürtel von Baselland über die Innerschweiz bis in nördliche Gebiete Graubündens erstreckt. Noch deutlicher sichtbar wird dieser Sachverhalt allerdings, wenn man sich auf diejenigen Ortspunkte beschränkt, bei welchen mehr als $75 \%$ die Variante (1) als natürlichste bezeich- 
nen. ${ }^{7}$ Man erhält so ein eigentliches Kerngebiet, wo die Verdoppelung des indefiniten Artikels sehr häufig als natürlichste Variante bezeichnet wird:

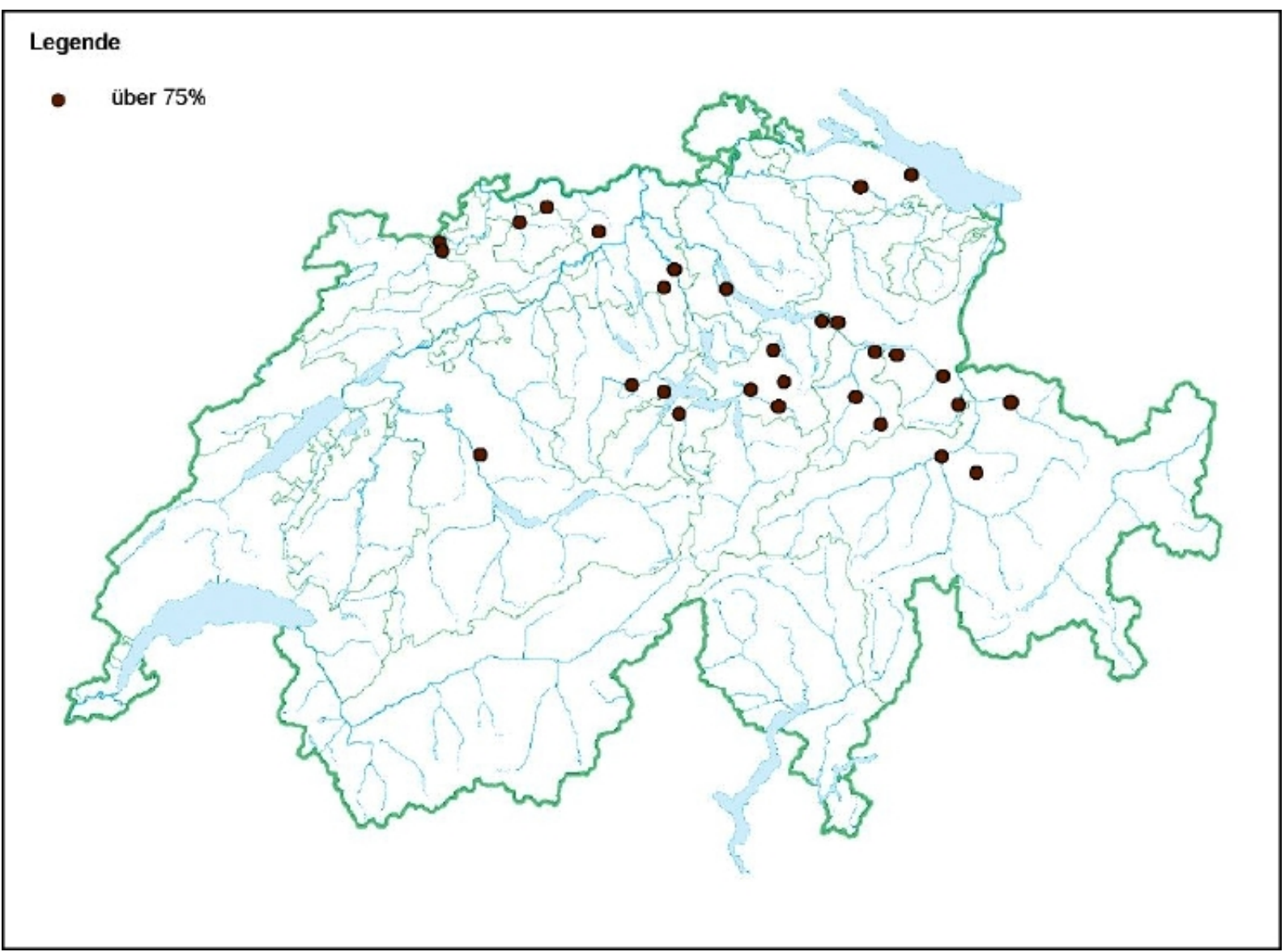

Abb. 5: "Also d' Susi wär e ganz e liebi Frau für de Markus!" - natürlichste Variante über 75\%

Bis jetzt haben wir uns diejenigen Gebiete angeschaut, welche die Verdoppelung akzeptieren oder sogar als natürlichste Variante bezeichnen. Das Gegenstück dazu, Gebiete, in welchen die Gewährsleute die Verdoppelung ablehnen, befindet sich im Wallis, Kanton Bern und Graubünden, vereinzelt auch in der Nordostschweiz. Die folgende Karte zeigt diejenigen Ortspunkte, bei denen mehr als 80\% der Befragten die Variante mit der Verdoppelung des indefiniten Artikels ablehnt. Einzelnennungen wurden wiederum herausgefiltert, damit diese das Bild nicht verfälschen.

\footnotetext{
${ }^{7}$ Auch bei dieser Karte sind diejenigen Orte, an welchen nur eine Person geantwortet hat, herausgefiltert.
} 


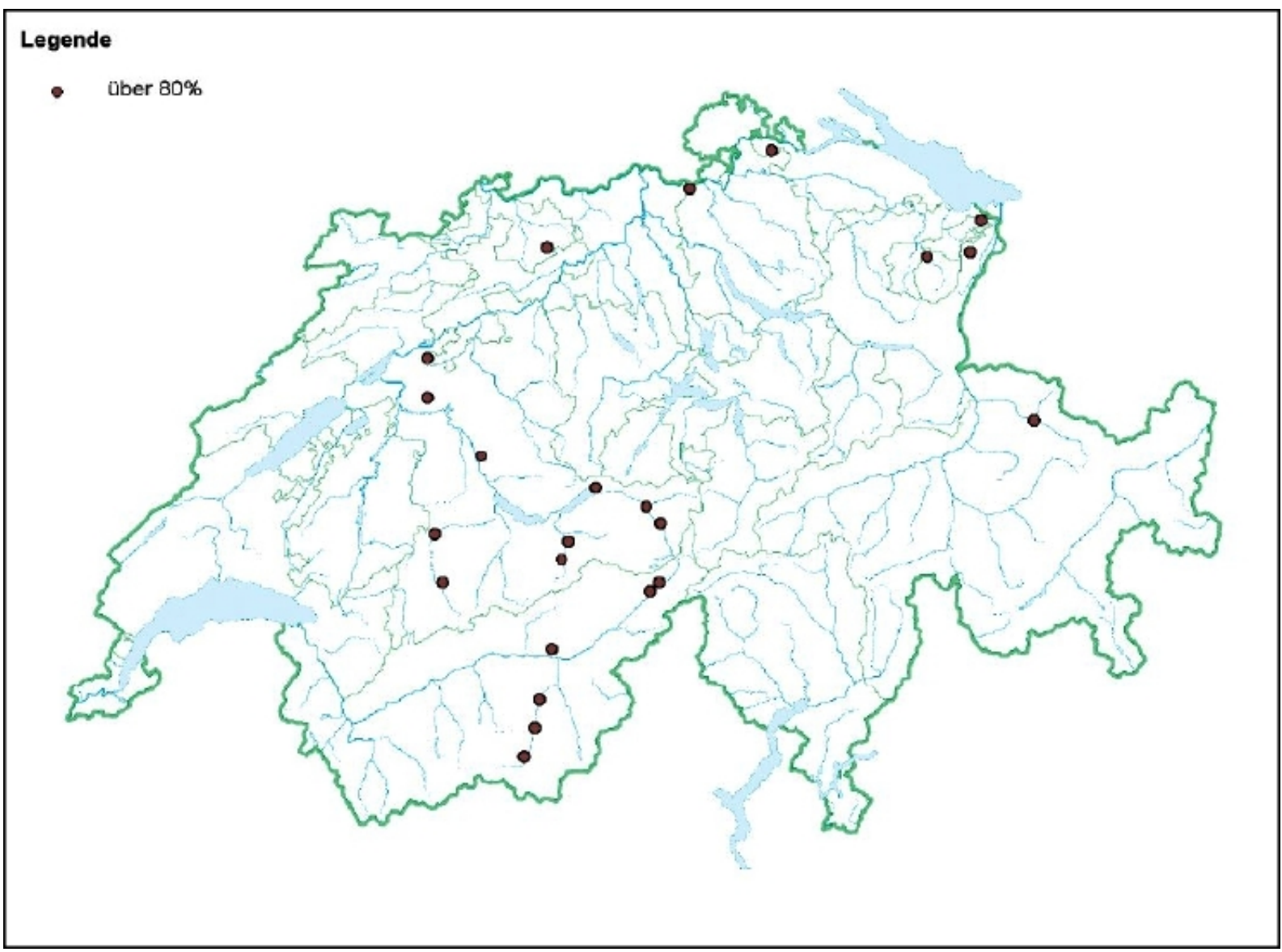

Abb. 6: "Also d' Susi wär e ganz e liebi Frau für de Markus!" - abgelehnt über $80 \%$

Es handelt sich vor allem um Ortspunkte der Kantone Bern und Wallis. Bei einem doch recht hohen Prozentsatz von $80 \%$ wage ich zu behaupten, dass an diesen Ortspunkten die Konstruktion mit dem verdoppelten Artikel kaum verwendet werden kann. Diese Tatsache wird noch dadurch verstärkt, dass 13 (über die Hälfte) der oben abgebildeten Ortspunkte die Verdoppelung sogar zu 100\% ablehnen. Ebenfalls spricht für diese These, dass nur gerade 6 Personen, welche an diesen Ortspunkten leben, die Variante (1) als natürlichste bezeichnen. Es gibt also ein kleines Gebiet von rund 20 Ortspunkten vor allem im Wallis und im Kanton Bern, an welchen die erweiterte Nominalphrase mit einem verdoppelten Artikel kaum bis gar nicht gebräuchlich ist.

Naheliegend wäre es nun zu glauben, dass ebenfalls ein Gebiet existiert, in welchem die Konstruktion mit der Verdoppelung weitgehend obligatorisch ist. Dazu müsste in der Versuchsanordnung die Variante (1) von den Gewährsleuten zu mindestens $80 \%$ bejaht, die anderen suggerierten Varianten jedoch verneint sein. Ebenfalls müsste die Variante (1) präferiert worden sein. Diese Abfrage ergibt folgendes Ergebnis: 


\begin{tabular}{|l|c|c|c|l|}
\hline Ortspunkt & Kanton & Anzahl GPn & $\begin{array}{l}\text { Variante (1): JA / Natürlichstes: 1 } \\
\text { anderes abgelehnt }\end{array}$ & $\%$ \\
\hline Einsiedeln & SZ & 6 & 6 & 100 \\
Maisprach & BL & 2 & 2 & 100 \\
Benken & SG & 5 & 5 & 100 \\
Boswil & AG & 2 & 2 & 100 \\
\hline
\end{tabular}

Abb. 7: "Also d' Susi wär e ganz e liebi Frau für de Markus!" - akzeptiert und präferiert über $80 \%$

Es gibt nur vier Ortspunkte, an denen die Gewährsleute die Verdoppelung über $80 \%$ akzeptieren $^{8}$ und die anderen Varianten ablehnen. Diese Ortschaften befinden sich alle in verschiedenen Kantonen. Man kann daher in keiner Weise von einem Gebiet sprechen, in welchem die Verdoppelung des indefiniten Artikels obligatorisch ist. Dazu kommt, dass an zwei der oben genannten Ortspunkten nur jeweils zwei Gewährsleute an der Untersuchung beteiligt waren, und dass deshalb dieses Ergebnis nicht sehr aussagekräftig ist. Übrig bleiben die beiden Ortschaften Einsiedeln (SZ) und Benken (SG) mit 6 resp. 5 Gewährspersonen. Dass Einsiedeln hier erscheint, deckt sich mit obigen Erkenntnissen aus den Abbildungen 3 und 5, wo gezeigt wurde, dass der Kanton Schwyz zum eigentlichen Kerngebiet der Verdoppelung gehört. Der Ortspunkt Benken liegt geographisch an der Kantonsgrenze zwischen dem Kanton St. Gallen und Schwyz und kann daher ebenfalls zu diesem Kerngebiet gezählt werden.

Ein Gebiet, in welchem die erweiterte Nominalphrase obligatorisch mit verdoppeltem indefiniten Artikel konstruiert wird, gibt es also nicht. Auch in Regionen, in welchen die Variante mit der Verdoppelung als häufigste und natürlichste bezeichnet wird, sind andere Konstruktionen möglich. Der Gebrauch der Konstruktion mit dem verdoppelten indefiniten Artikel ist demzufolge optional.

Wovon hängt jedoch diese Variation ab? Wann konstruiert ein Sprecher oder eine Sprecherin einen Satz mit verdoppeltem Artikel und wann nicht? Im nächsten Kapitel werde ich aufführen, welche intra- und extralinguistischen Faktoren für diese Variation verantwortlich sein könnten.

\section{$6 \quad$ Variation}

Wie oben bereits erwähnt, besteht der nächste Schritt darin, sowohl extralinguistischen als auch intralinguistischen Parametern nachzugehen, welche die Konstruktion der adverbiell erweiterten Nominalphrase beeinflussen können. ${ }^{9}$

\footnotetext{
${ }^{8}$ Es ist Zufall, dass an allen vier Ortspunkten $100 \%$ der Befragten auf diese Weise geantwortet haben. Meine Abfrage suchte lediglich nach solchen, bei welchen über $80 \%$ der Gewährsleute die Suchkriterien erfüllen. Daraus lässt sich schliessen, dass es keine Ortspunkte gibt, deren Resultate auf diese Abfrage zwischen 80 und $100 \%$ liegen.

${ }^{9}$ Für detailliertere Erläuterungen zu den Parametern und Begründungen, weshalb diese Teil der Untersuchung sind, cf. Steiner (2002).
} 
Extralinguistische Parameter

- Alter der Gewährsperson

- Geschlecht der Gewährsperson

- Soziale Schicht der Gewährsperson

- Sprachraum ${ }^{10}$

\section{Intralinguistische Parameter}

- Syntaktische Konstruktion (Satzart, Kopfstellung/Schlussstellung im Satz etc.)

- Semantik

- Suprasegmentalia (Rhythmus, Silbenmenge)

Der Rahmen dieses Beitrages sowie der gegenwärtige Stand meiner Forschung erlauben es mir nicht, auf alle obigen Punkte einzugehen. Ich werde mich im Folgenden darauf beschränken, den Einfluss der extralinguistischen Parameter Alter, Geschlecht und soziale Schicht zu erläutern. Indem ich den Einfluss des Parameters Alter auf den Forschungsgegenstand untersuche, erhalte ich eine sogenannte apparent time analysis.

\section{$7 \quad$ Diachrone Betrachtung (apparent time analysis)}

Analysiert man die Daten aus obiger Versuchsanordnung nun quantitativ unter Berücksichtigung des Alters der Gewährspersonen, so ergibt sich die folgende Verteilung:

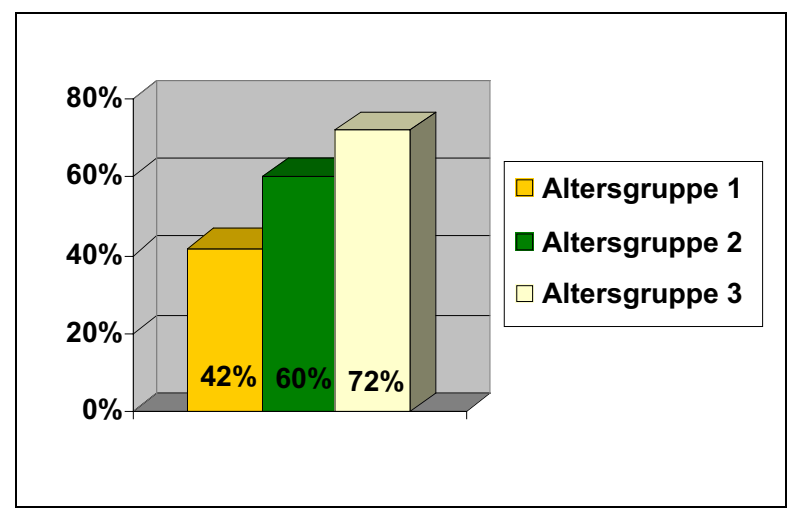

Abb. 8: Akzeptanz der Variante (1) bei Altersgruppe 1-311

Hier sieht man ganz deutlich, dass die Variante (1) bei den jüngeren Gewährspersonen an Akzeptanz gewonnen hat. Die Konstruktion mit der Verdoppelung des indefiniten Artikels ist bei den jüngeren Gewährsleuten deutlich weiter verbreitet als bei den älteren.

Bei der Variante (2) ergeben sich keine relevanten Unterschiede in der Akzeptanz zwischen den verschiedenen Altersgruppen. ${ }^{12}$ Ich wage zu behaupten, dass die Variante (2) unabhängig vom Alter der Gewährsleute konstant mit einer hohen Rate akzeptiert wird.

\footnotetext{
${ }^{10}$ Der Einfluss dieses Parameters auf den Forschungsgegenstand wurde im Kapitel 5 des vorliegenden Beitrages bereits erläutert.

${ }^{11}$ Bei Altersgruppe 1 handelt es sich um die ältesten Gewährpersonen (Jahrgang 1927 und älter), bei Altersgruppe 3 um die jüngsten (Jahrgang 1958 und jünger). Detaillierte Ausführungen zur Einteilung der Datenserien finden sich in Steiner (2002: 18ff.).

${ }^{12}$ Für detaillierte Ausführungen hierzu cf. Steiner (2002: 23).
} 
Die Ergebnisse der Variante (3) ergeben folgende Abbildung:

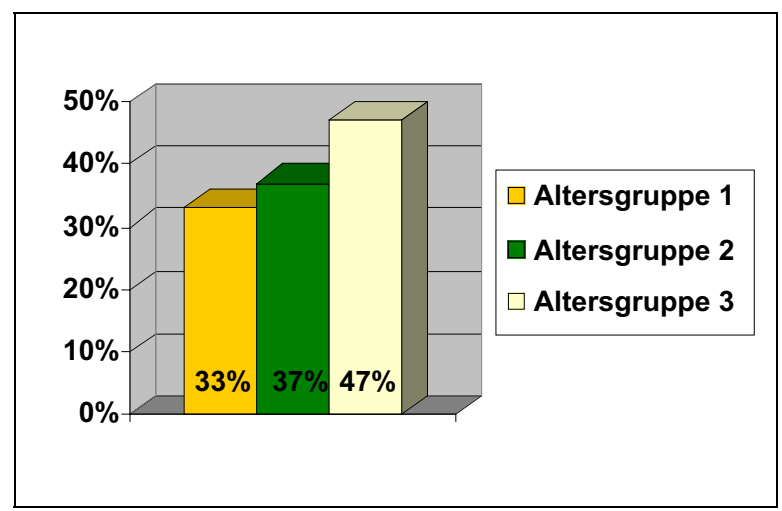

Abb. 9: Akzeptanz der Variante (3) bei Altersgruppe 1-3

Wie schon bei der Variante (1) zeichnet sich auch hier eine Zunahme der Akzeptanz von Variante (3) ab.

Bei einem Vergleich mit den Antworten zur natürlichsten Variante (Präferenz) wird der Befund sowohl für die Variante (1) als auch für Variante (2) erhärtet. Die Variante (3) jedoch, welche bei der Akzeptanz eine Zunahme bei den jüngeren Gewährspersonen verzeichnete, zeigt bei der Präferenz eine gegenteilige Entwicklung:

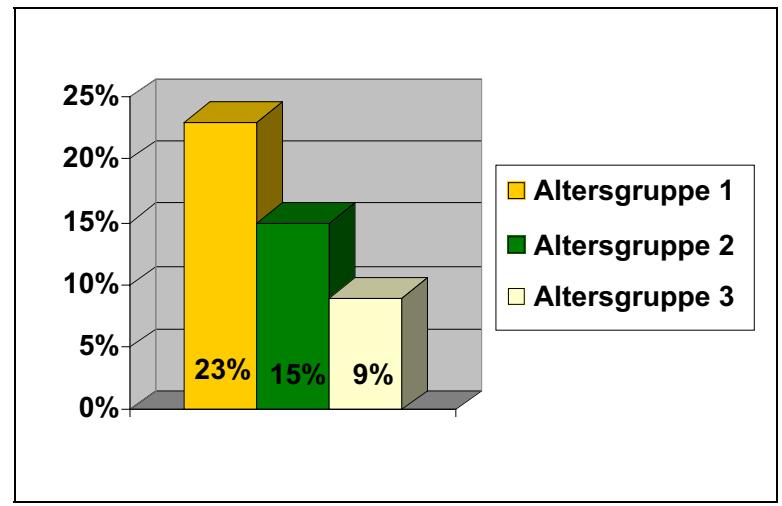

Abb. 10: Variante (3) als natürlichste bei Altersgruppe 1-3

Man sieht sehr schön, dass die Variante (3) bei der älteren Generation recht häufig präferiert wird, während sie bei der Altersgruppe 2 von deutlich weniger Gewährspersonen genannt wird. Diese Entwicklung wird bei Altersgruppe 3 fortgesetzt; bei den Gewährsleuten dieser Gruppe wird die Variante (3) noch weniger häufig präferiert. Dies könnte ein Hinweis darauf sein, dass die Variante (3) bei den älteren Leuten noch bekannt ist und auch verwendet wird, während sie die jüngeren Leute, obwohl sie sie kennen und akzeptieren, kaum präferieren.

Zusammenfassend lässt sich festhalten, dass der Parameter Alter einen grossen Einfluss auf die Konstruktion der adverbiell erweiterten Nominalphrase ausübt. So wird die Variante mit der Artikelverdoppelung von den jüngeren Gewährspersonen zu einem deutlich höheren Prozentsatz akzeptiert und auch präferiert als dies bei den älteren Personen der Fall ist. Auch die Variante (3) mit vorangestelltem Artikel wird von den verschiedenen Altersgruppen unterschiedlich akzeptiert und präferiert. 


\section{$8 \quad$ Einfluss des Parameters Geschlecht}

Um zu prüfen, ob und inwiefern der Faktor Geschlecht einen Einfluss auf die adverbiell erweiterte indefinite Nominalphrase ausübt, wurde wiederum dieselbe Versuchsanordnung quantitativ ausgewertet, diesmal jedoch unter Berücksichtigung des Geschlechts der Gewährspersonen. Dies ergibt folgendes Bild:

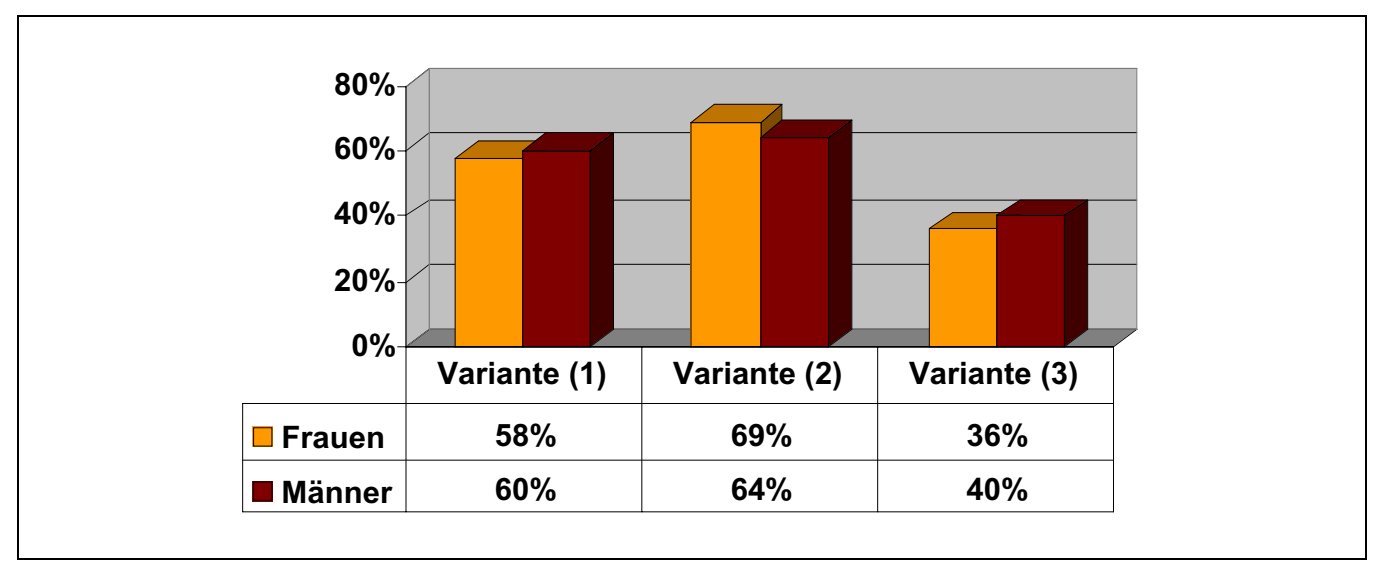

Abb. 11: Akzeptanz der Varianten 1-3 bei Frauen und Männern

Entgegen meinen Erwartungen ergeben sich fast keine Unterschiede in der Akzeptanz der verschiedenen Varianten bei Frauen und Männern. Die Variante (2) wird von beiden Geschlechtern am meisten akzeptiert, während die Variante (1) bei beiden etwas weniger häufig angekreuzt wurde. Die Variante (3) ist sowohl bei den Frauen als auch bei den Männern deutlich die am schlechtesten akzeptierte Möglichkeit. Die Prozentzahlen unterscheiden sich bei den Geschlechtern nur um 2-5\%, was m. E. ein nicht sehr aussagekräftiger Unterschied ist.

Der einzige Unterschied, der sich im Hinblick auf die Geschlechter feststellen lässt, ist, dass die Männer deutlich weniger grosse Unterschiede zwischen den einzelnen Varianten machen als die Frauen. Dies ist in der folgenden Abbildung noch klarer ersichtlich.

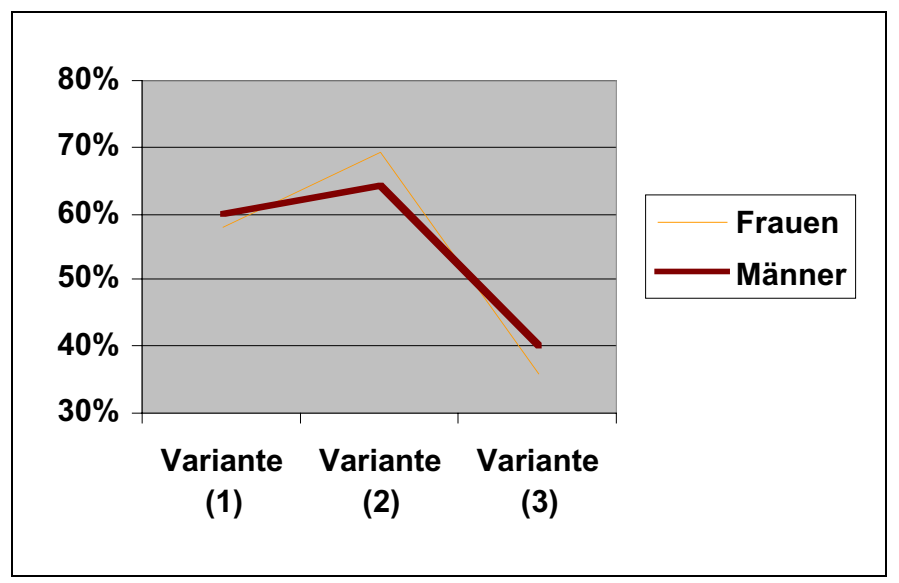

Abb. 12: Akzeptanz der Varianten 1-3 bei Frauen und Männern 
Man sieht hier, dass die Frauen grössere Unterschiede in der Akzeptanz der verschiedenen Varianten aufweisen, als dies die Männer tun. Der Graph der Männer im obigen Diagramm ist daher etwas flacher als derjenige der Frauen.

Zusammenfassend lässt sich jedoch sagen, dass der Faktor Geschlecht praktisch keinen Einfluss auf die Akzeptanz der verschiedenen Varianten ausübt. Übt er jedoch auch auf die Wahl der natürlichsten Variante, also auf die Präferenz, keinen Einfluss aus? Folgende Abbildung stellt die Verteilung der präferierten Variante nach Geschlechtern getrennt dar:

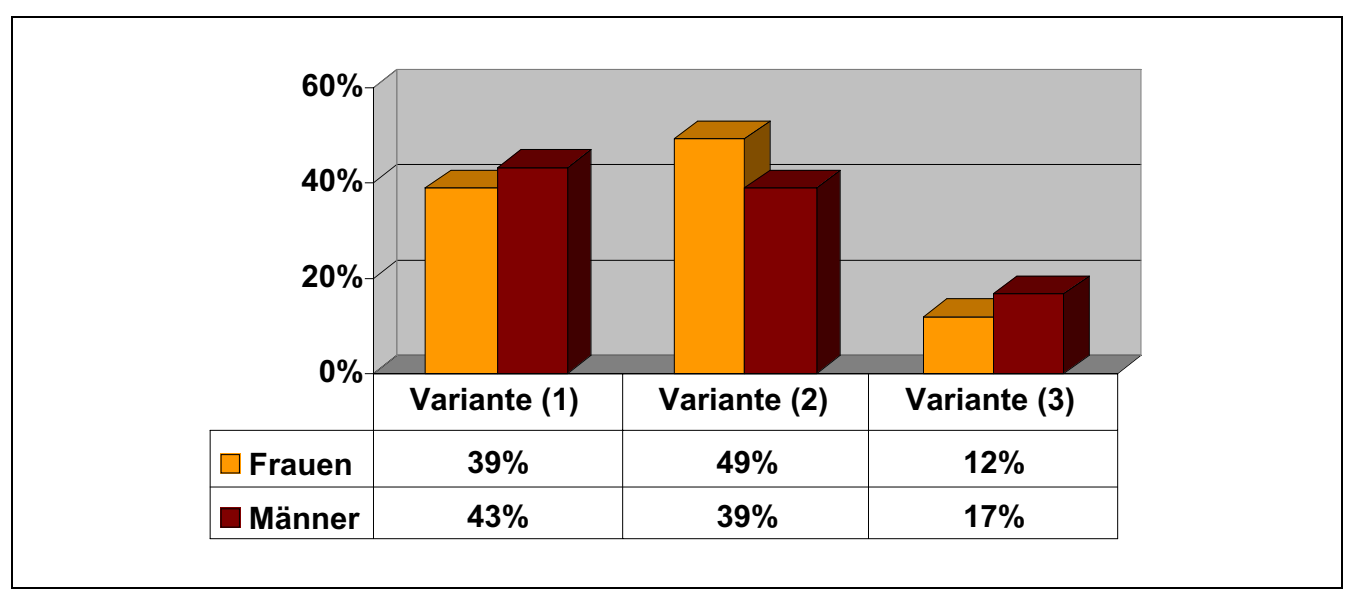

Abb. 13: Natürlichste Variante bei Frauen und Männern

Hier lassen sich etwas grössere Unterschiede feststellen als bei der Akzeptanz. Bei der Variante (2) unterscheidet sich der Wert bei den Frauen und Männern um 10\%. Die Frauen bevorzugen als natürlichste Variante eindeutig die zweite, während die Männer die erste der zweiten (wenn auch geringfügig) vorziehen. Dies bewirkt die relativ grosse Differenz zwischen den Prozentzahlen bei Variante (2). Bei den Varianten (1) bzw. (3) liegt der prozentuale Unterschied bei 4 respektive 5\%. In Kurven dargestellt sieht man wiederum deutlich, dass die Gruppe der Frauen klarer eine Variante favorisiert als die Gruppe der Männer; bei den Männern ergibt sich wiederum ein flacherer Graph als bei den Frauen:

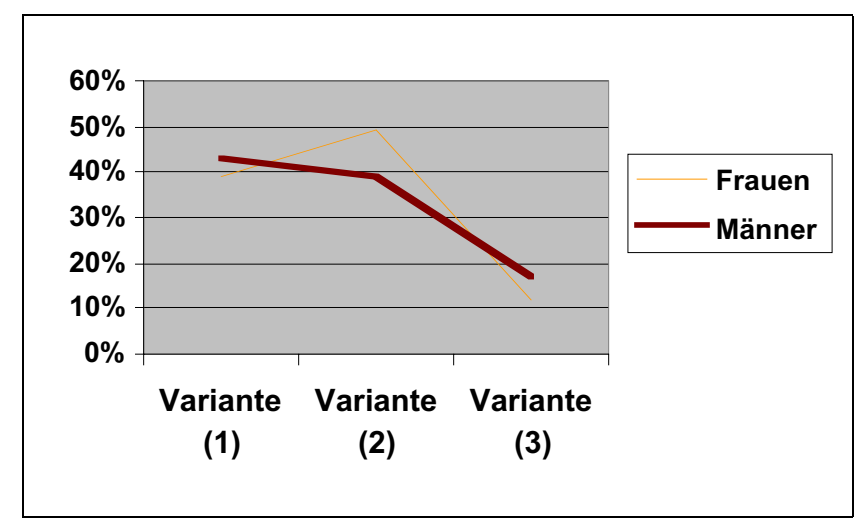

Abb. 14: Natürlichste Variante bei Frauen und Männern

Zusammenfassend lässt sich festhalten, dass die Gruppe der Männer tendenziell sowohl bei der Akzeptanz der Varianten als auch bei der Präferenz "toleranter" sind. Das heisst, sie haben keinen Spitzenreiter, der mit grossem Abstand heraussticht, wie dies die Frauen haben. Für 
die Gruppe der Frauen heisst das, dass sie eine klarer favorisierte Variante haben und weniger "tolerant" gegenüber den anderen Möglichkeiten sind.

Die Männer nennen als natürlichste Variante am häufigsten die Variante (1), während die Frauen die Variante (2) wählen.

In einem nächsten Schritt wäre nun zu prüfen, inwiefern eine Interdependenz zwischen dem Parameter Alter und dem Parameter Geschlecht besteht.

\section{$9 \quad$ Einfluss des Parameters soziale Schicht}

Im ersten Fragebogen des Nationalfondsprojektes zur Dialektsyntax wurde von allen Gewährspersonen auch der Beruf erfragt. Aufgrund dieser Berufsbezeichnungen habe ich eine Einteilung in Gruppen vorgenommen. ${ }^{13}$ Bei den Bezeichnungen der Schichten habe ich mich an Labovs großangelegter Studie "Social Stratification of English in New York City" (1966) orientiert. Somit ergibt sich die folgende Einteilung:

\begin{tabular}{|l|l|}
\hline Code & Bezeichnung \\
\hline 1 & upper class \\
\hline 2 & lower middle class \\
\hline 3 & working class \\
\hline
\end{tabular}

Die Akzeptanz der drei möglichen Konstruktionsweisen sieht bei den einzelnen sozialen Schichten folgendermassen aus:

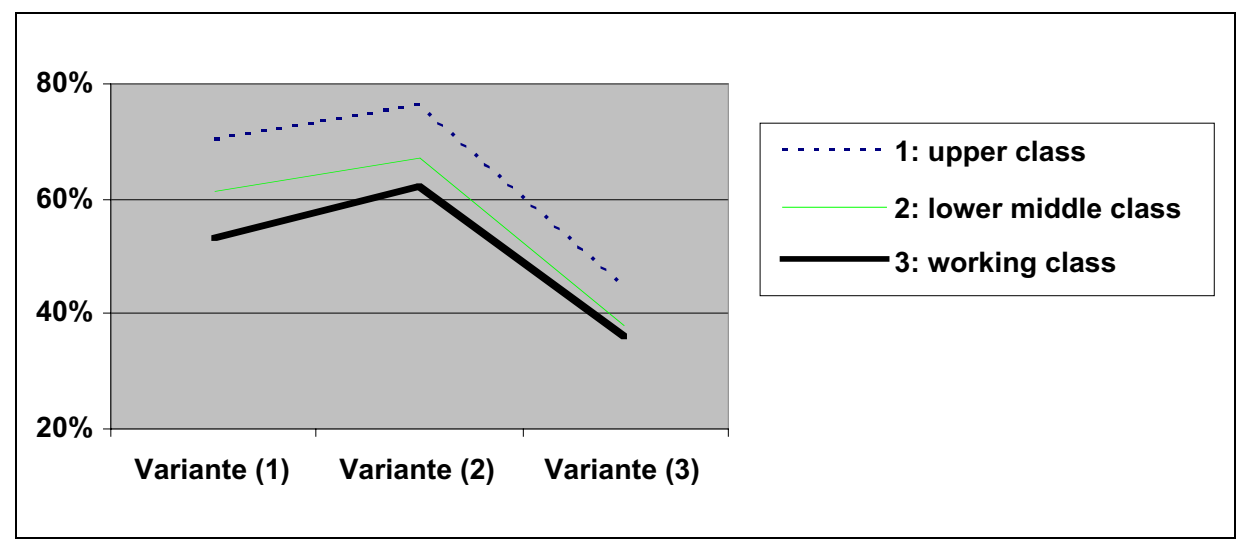

Abb. 15: Akzeptanz der Variante 1-3 bei den Gruppen 1-3

Diese Abbildung zeigt ganz deutlich, dass alle drei Schichten die verschiedenen Varianten praktisch identisch akzeptieren. Bei allen Schichten ist die Variante (2) am häufigsten akzeptiert, gefolgt von Variante (1), während die Variante (3) bei allen deutlich am wenigsten akzeptiert wird. Der einzige Unterschied, der sich feststellen lässt, liegt darin, dass die Prozentzahlen von der upper class über die lower middle class zu der working class sinken. Die relativ hohen Prozentwerte der upper class weisen darauf hin, dass sehr viele Gewährspersonen mehrere Varianten angekreuzt haben. Diese Mehrfachakzeptanz bewirkt einen Anstieg

\footnotetext{
13 Detaillierte Erläuterungen zu der Einteilung in soziale Schichten finden sich in Steiner (2002: 44f.)
} 
des Prozentwertes und eine grosse Überschreitung des 100\%-Werts. Die upper class weist demnach eine breite Akzeptanz aller drei Varianten auf. Die working class hingegen hat kleinere Prozentzahlen, liegt näher bei der 100\%-Marke und hat daher auch weniger Mehrfachakzeptanzen als die upper class. Die working class hat also eine weniger breite Akzeptanz, das heisst die Gewährspersonen entscheiden sich tendenziell eher für eine einzelne Variante als mehrere anzukreuzen.

Aufgrund dieser Ergebnisse ist nun zu erwarten, dass sich bei der Präferenz praktisch keine Unterschiede zwischen den Schichten ergeben. Bei der Frage nach der natürlichsten Variante fallen nämlich praktisch alle Mehrfachakzeptanzen weg, da die Gewährspersonen gezwungen werden, sich für eine Variante zu entscheiden. Da die Mehrfachakzeptanzen bis hierher als einziger Verursacher für die unterschiedlichen Ergebnisse der Schichten verantwortlich gemacht werden können, ist nun zu erwarten, dass die Unterschiede ausbleiben. Dies wird durch folgendes Diagramm bestätigt:

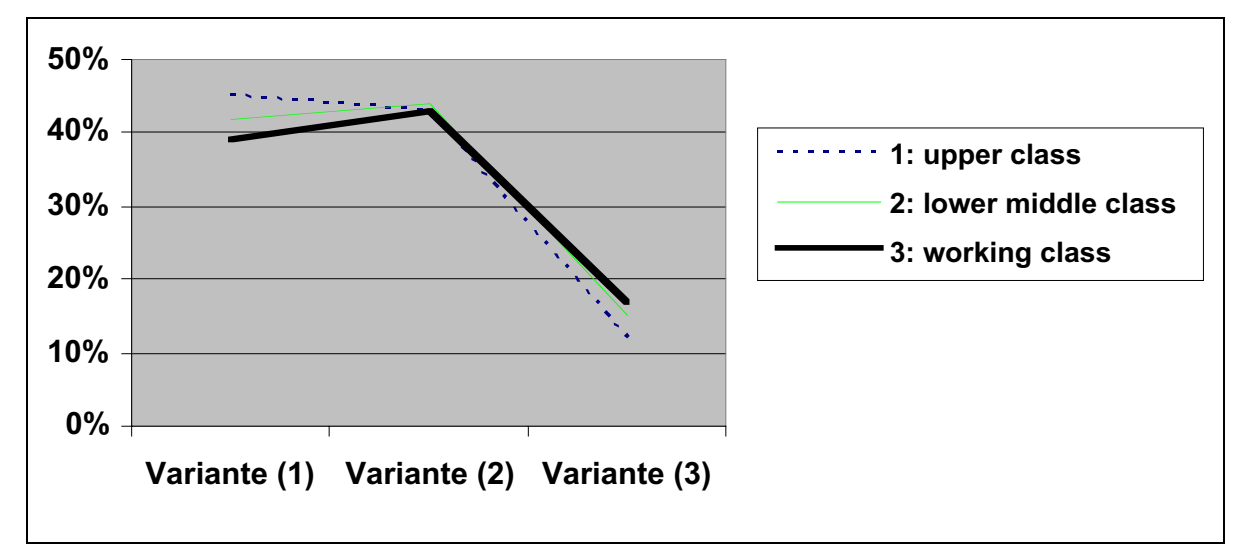

Abb. 16: Natürlichste Variante 1-3 bei den Gruppen 1-3

Die Graphen der verschiedenen sozialen Schichten sind fast deckungsgleich. Bei allen Schichten wird die Variante (3) am wenigsten präferiert. Bei der working class und der lower middle class wird zudem die Variante (2) am häufigsten bevorzugt. Einzig die upper class bevorzugt die Variante (1) am meisten.

Abschliessend kann ich sagen, dass die soziale Schicht kaum Einfluss auf die adverbiell erweiterte indefinite Nominalphrase ausübt. Die Akzeptanzen der einen oder anderen Variante liegen bei allen drei Schichten gleich verteilt.

\section{Zusammenfassung und Ausblick}

Bei einer Abfrage der Akzeptanz der drei möglichen Konstruktionsweisen durch MultipleChoice-Verfahren findet die Variante mit dem Artikel in der Zwischenstellung bei den Gewährsleuten den grössten Zuspruch, gefolgt von der Konstruktion mit verdoppeltem Artikel und dann derjenigen mit vorangestelltem Artikel. Die Frage nach der natürlichsten Variante (Präferenz) wurde von den Gewährsleuten dahingehend beantwortet, dass die Variante mit dem verdoppelten Artikel in etwa gleich häufig präferiert wird wie diejenige mit dazwischen- 
gestelltem Artikel. Die Konstruktion mit dem vorangestellten Artikel ist eher selten präferiert worden.

In geographischer Hinsicht gibt es zwei Kantone, welche die Verdoppelung ablehnen. Das sind die Kantone Bern und Wallis. Hingegen kann man ein Kerngebiet von Baselland über die Zentralschweiz bis in den Norden Graubündens lokalisieren, in welchem die Verdoppelung aussergewöhnlich häufig akzeptiert und auch als natürlichste Variante bezeichnet wird. Es gibt jedoch kein Gebiet, in welchem die Verdoppelung obligatorisch wäre. Es handelt sich also um fakultative Variation, welche zusätzlich von anderen Faktoren beeinflusst wird.

Das Alter der Gewährsperson hat einen grossen Einfluss auf die Konstruktion der adverbiell erweiterten indefiniten Nominalphrase. Die Akzeptanz der Variante mit der Artikelverdoppelung steigt enorm von den älteren zu den jüngeren Gewährspersonen. Man kann davon ausgehen, dass sich die Akzeptanz im Laufe der Zeit erhöht hat. Auch die Präferenz der Variante mit Verdoppelung hat zugenommen und ist bei den jüngeren Gewährspersonen quasi gleich hoch wie diejenige der Variante mit dem Artikel in der Zwischenstellung.

Auf Letztere hingegen hat das Alter der Gewährsperson kaum einen Einfluss. Die Ergebnisse sind über alle drei Altersgruppen ähnlich.

Die Variante (3) mit dem vorangestellten Artikel gewinnt bei den jüngeren Gewährspersonen an Akzeptanz, wird jedoch seltener präferiert.

Auch das Geschlecht der Gewährsperson scheint einen gewissen Einfluss auf die Konstruktion zu haben. Die männlichen Gewährspersonen machen sowohl bei der Akzeptanz als auch bei der Präferenz deutlich weniger grosse Unterschiede zwischen den Konstruktionsmöglichkeiten. Die weiblichen Gewährsleute hingegen haben jeweils eine klar favorisierte Variante. Ebenso bezeichnet die Gruppe der Männer die Variante (1) mit dem verdoppelten Indefinitartikel als natürlichste, während die Gruppe der Frauen die zweite Variante mit dem zwischengestellten Artikel bevorzugen.

Die soziale Schicht der SprecherInnen scheint jedoch kaum Einfluss auf die Konstruktionsart der adverbiell erweiterten indefiniten Nominalphrase zu haben.

In einem weiteren Schritt meiner Forschung werde ich untersuchen, ob und wie oft Gewährsleute auch aktiv Konstruktionen mit verdoppeltem Artikel produzieren. Grundlage hierfür werden im Jahr 2002 schriftlich erhobenes, bis jetzt noch nicht ausgewertetes Datenmaterial, sowie mündliche Belege sein. Bei oberflächlicher Sichtung der Daten konnte ich bereits feststellen, dass die Gewährsleute im Vergleich zur Akzeptanz von suggerierten Varianten erstaunlich wenige Verdoppelungen produzieren. Dieses Vorergebnis werde ich wiederum mit den Daten des Nationalfondsprojektes Dialektsyntax des Schweizerdeutschen überprüfen können, da dort im vierten Fragebogen eine Übersetzungsfrage zum besagten Phänomen enthalten ist. Der vierte Fragebogen wird momentan noch erfasst und ist daher noch nicht ausgewertet worden. 
Die Semantik der Konstruktion ist ein weiterer Bereich, der für die weiterführende Untersuchung unerlässlich ist. Der Hinweis auf semantische Unterschiede wurde auch immer wieder von Gewährspersonen gemacht.

Dem Bereich der Suprasegmentalia werde ich mich in der weiteren Forschung zum Thema ebenfalls widmen.

\section{Literaturangaben}

Binz, Gustav (1888): Zur Syntax der baseldeutschen Mundart. Stuttgart.

Bossard, Hans (1962): Zuger Mundartbuch. Zürich.

Bucheli Berger, Claudia (2005): "Passiv im Schweizerdeutschen". Linguistik online 24, 3/05: 49-77. http://www.linguistik-online.de/24_05/bucheli.pdf

Bucheli Berger, Claudia/Glaser, Elvira (2002): "The syntactic Atlas of Swiss German Dialects: empirical and methodological problems". In: Barbiers, Sjef/Cornips, Leonie/van der Kleij, Susanne (eds.): Syntactic Microvariation. Amsterdam: 42-74. http://www.meertens.knw.nl/books/synmic/.

Christen, Helen (2003): "Uu fein, welts gut und rüüdig schöön - Überlegungen zu lexikalischen Aspekten eines SchweizerDeutsch der Regionen". In: Dittli, Beat/Häcki Buhofer, Annelies/Haas Walter (eds.): Gömmer MiGro? Veränderungen und Entwicklungen im heutigen SchweizerDeutschen. Freiburg/Schweiz: 25-38.

Fischer, Ludwig (1960): Luzerndeutsche Grammatik. Zürich.

Frey, Arthur (1906): "Beiträge zur Syntax des Schweizerischen". In: Glock, Anton et al. (eds.): Analecta Germanica. Hermann Paul zum 7. August 1906. Amberg: 19-42.

Henn-Memmesheimer, Beate (1986): Nonstandardmuster. Ihre Beschreibung in der Syntax und das Problem ihrer Arealität. Tübingen.

Hodler, Werner (1969): Berndeutsche Syntax. Bern.

Hotzenköcherle, Rudolf (ed.) (1975): SDS: Sprachatlas der deutschen Schweiz. III. Bd: Formengeographie. Bern.

Labov, William (1966): The Social Stratification of English in New York City. Washington D.C.

Lakoff, Robin (1973): "Language and women's place". Language and Society 2: 45-80.

Marti, Werner (1985): Berndeutsch-Grammatik. Bern.

Plank, Frans (2003): "Double Articulation". In: Plank, Frans (ed.) Noun Phrase Structure in the Languages of Europe. Berlin: 337-395.

Schweizerisches Idiotikon (1881ff.): Wörterbuch der schweizerdeutschen Sprache. Frauenfeld.

Stalder, Franz Joseph/Bigler, Niklaus (ed.) (1994, 1806): Schweizerisches Idiotikon. Mit etymologischen Bemerkungen untermischt. Samt einem Anhange der verkürzten Taufnamen. Aarau. Stark umgestalt. u. erw. Bearb. der Publikation 1806/12 "Versuch eines Schweizerischen Idiotikons." (= Reihe Sprachlandschaft 14). 
Steiner, Janine (2002): E ganz e gueti Frau - Zur Verdoppelung des indefiniten Artikels in der adverbiell erweiterten Nominalphrase im Schweizerdeutschen. Unpublizierte Lizentiatsarbeit an der Universität Zürich.

Stucki, Karl (1921): Schweizerdeutsch. Abriss einer Grammatik mit Laut-und Formenlehre. Zürich.

Suter, Rudolf (1976): Baseldeutsch-Grammatik. Basel.

Weber, Albert (1948): Zürichdeutsche Grammatik. Zürich.

Weise, Oskar (1909): "Der gegenwärtige Stand der Forschung auf dem Gebiete der Syntax deutscher Mundarten". In: Schröder, Heinrich (ed.): Germanisch-romanische Monatsschrift 1. Kiel: 733-742. 